\title{
含苯并三氮唑单元的新型吡唑肟衍生物的合成与生物活性
}

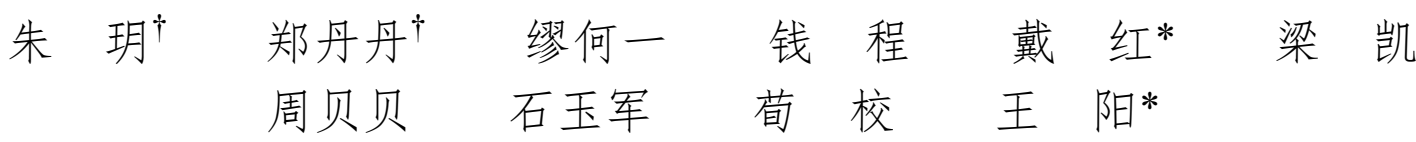 \\ (南通大学化学化工学院 南通 226019)
}

\begin{abstract}
摘要 为了探寻新型含氮杂环活性物质, 以杀螨剂唑螨酯为先导化合物, 在吡唑肜的分子结构中引入苯并三氮唑单元 结构, 设计合成了 17 个未见文献报道的含苯并三氮唑结构的新型吡唑肜类衍生物. 目标化合物的结构经 ${ }^{1} \mathrm{H} \mathrm{NMR}^{13} \mathrm{C}$ NMR 和元素分析确证. 初步的生测数据表明, 部分目标化合物显示出优良杀虫作用. 在 $20 \mu \mathrm{g} / \mathrm{mL}$ 浓度下, 3 个化合物对 朱砂叶螨有 $70 \% \sim 100 \%$ 的防效. 6 个化合物在 $20 \mu \mathrm{g} / \mathrm{mL}$ 浓度下对蚜虫的杀死率在 $90 \% \sim 100 \%$. 另外, 16 个化合物在 500 $\mu \mathrm{g} / \mathrm{mL}$ 浓度下对粘虫也呈现出较好的杀虫活性(致死率 $\geqslant 80 \%$ ).
\end{abstract}

关键词 苯并三氮唑; 吡唑肜; 合成; 生物活性

\section{Synthesis and Biological Activities of Novel Pyrazole Oxime Derivatives Containing Benzotriazolyl Moiety}

\author{
Zhu, Yue ${ }^{\dagger}$ \\ Zheng, Dandan ${ }^{\dagger}$ \\ Miao, Heyi \\ Qian, Cheng \\ Dai, Hong* \\ Liang, Kai \\ Zhou, Beibei \\ Shi, Yujun \\ Xun, Xiao \\ Wang, Yang* \\ (College of Chemistry and Chemical Engineering, Nantong University, Nantong 226019)
}

\begin{abstract}
In search of new N-heterocyclic compounds possessing good biological activities, 17 novel pyrazole oxime derivatives were designed and synthesized by introducing benzotriazole moiety into pyrazole oxime skeleton based on fenpyroximate. Their structures were tested by ${ }^{1} \mathrm{H}$ NMR, ${ }^{13} \mathrm{C}$ NMR and elemental analysis. Preliminary bioassay data displayed that 3 compounds showed $70 \% \sim 100 \%$ insecticidal activities towards Tetranychus cinnabarinus at $20 \mu \mathrm{g} / \mathrm{mL}$. 6 compounds possessed $90 \% \sim 100 \%$ mortality rates towards Aphis medicaginis at $20 \mu \mathrm{g} / \mathrm{mL}$. Moreover, 16 compounds displayed more than $80 \%$ insecticidal activities against Mythimna separata (Walker) at $500 \mu \mathrm{g} / \mathrm{mL}$.

Keywords benzotriazole; pyrazole oxime; synthesis; biological activity
\end{abstract}

目前, 含氮杂环单元因其具有多样的生物活性而成 为新药创制研究的热点领域 ${ }^{[1-10]}$. 苯并三氮唑为一种常 见的含氮杂环结构, 多用于杀菌、除草、抗病毒、抗癌 等生物活性分子研究中, 在植物保护和医疗保健等方面 有着广阔的应用 ${ }^{[11-16]}$. 如 Zhu 课题组 ${ }^{[17]}$ 报道的化合物 $\mathbf{A}$ 呈现出良好的杀菌效果, 化合物 $\mathbf{A}$ 在 $50 \mu \mathrm{g} / \mathrm{mL}$ 测试浓 度下对苹果轮纹病菌的防效达 $56.8 \%, \mathrm{He}$ 等 ${ }^{[18]}$ 研发的化 合物 B 具有除草与酩醇酸还原异构酶抑制活性. 吡唑肜 衍生物也是氮杂环中的一员, 展现出优异杀菌、杀虫及 抗癌等活性, 在农药与医药方面起到重要作用 ${ }^{[19-22]}$. 如
日本 Nihon Nohyaku 公司报道的杀螨剂唑螨酯(Fenpyroximate)对螨虫等害虫有显著的防治效果，唑螨酯由于 具有持效期较长、受季节影响小等特点 ${ }^{[23]}$ 引起药物化学 家的广泛关注. 近年来, 不少具有良好生物活性的吡唑 肟衍生物被成功研制出来, 如 Zou 课题组 ${ }^{[24]}$ 开发的含吡 啶基吡唑肜衍生物 $\mathbf{C}$ 对螨虫和蚜虫表现出良好的杀虫 效果，化合物 $\mathbf{C}$ 在 $100 \mu \mathrm{g} / \mathrm{mL}$ 测试浓度下对螨虫和蚜虫 的杀死率分别为 $100 \%$ 和 $90 \%$; 同时, 本课题组研究发 现含噁唑联苯基单元的吡唑肜 $\mathbf{D}^{[25]}$ 和含 1,3,4-噁二唑联 苯基结构的吡唑肜 $\mathbf{E}^{[26]}$ 呈现出较好的杀虫作用, 化合物

\footnotetext{
* Corresponding authors. E-mail: daihong_2015@aliyun.com; wangyang1617@sina.com Received August 18, 2020; revised September 27, 2020; published online October 13, 2020.

Project supported by the National Natural Science Foundation of China (Nos. 21372135, 21802075), and the Science and Technology Project Fund of Nantong City (No. MS12019060).

国家自然科学基金(Nos. 21372135, 21802075)、南通市科技计划(No. MS12019060)资助项目.

†共同第一作者(These authors contributed equally to this work).
} 
D 在 $500 \mu \mathrm{g} / \mathrm{mL}$ 测试浓度下对粘虫、螨虫和蚜虫的杀死 率分别为 $100 \%, 90 \%$ 和 $100 \%$, 化合物 $\mathbf{E}$ 在 $500 \mu \mathrm{g} / \mathrm{mL}$ 测试浓度下对粘虫与蚜虫的防效分别为 $100 \%$ 和 $80 \%$. 因此, 为了继续从吡唑肜类化合物中寻找出具有优良生 物活性的物质, 本工作在杀螨剂唑螨酯与前期研究基础 上，运用活性片段杂合策略，对唑螨酯中吡唑环 4-位取 代苯基部分进行改造, 将叔丁基酯基片段用苯并三氮唑 杂环替代，同时将唑螨酯中吡唑环 5-位的苯基用取代苯 环替代, 设计制备出了一系列含苯并三氮唑单元的新型 吡唑肟化合物, 并测定了所合成的化合物对朱砂叶螨、 粘虫和蚜虫的生物活性. 详细的合成路线见 Scheme 1.<smiles>O=C(OC(Cn1nnc2ccccc21)C(=O)c1ccccc1)c1cccnc1</smiles><smiles>Clc1ccc(CN2CCN(Cn3nnc4ccccc43)CC2)c(Cl)c1</smiles><smiles>Cc1nn(C)c(Oc2ccccc2)c1/C=N/OCc1ccc(C(=O)OC(C)(C)C)cc1/C=N/OCc1ccc(C(=O)OC(C)(C)C)cn1</smiles><smiles>Cc1nnc(-c2ccc(CO/N=C/c3c(C)nn(C)c3Oc3ccccc3)cc2)o1</smiles>

图 1 化合物 $\mathbf{A} \sim \mathbf{E}$ 和唑螨酯的结构

Figure 1 Structures of compounds $\mathbf{A} \sim \mathbf{E}$ and fenpyroximate

\section{1 结果与讨论}

\section{1 目标化合物 7 的合成}

在制备目标产物 7 时, 以 $7 \mathrm{~g}$ 为例, 尝试了不同的缚 酸剂如三乙胺、碳酸钠、碳酸钾、氢氧化钾, 不同的溶 剂如三氯甲烷、乙腈、 $N, N$-二甲基甲酰胺(DMF)对 $7 \mathbf{g}$ 单步收率的影响(表 1). 实验过程中发现, 选以碳酸钾为 缚酸剂, 以乙腈为溶剂, 加热回流反应 $10 \mathrm{~h}$, 反应转化 率较高, $7 \mathrm{~g}$ 的单步收率为 $70 \%$. 因此, 最后通过以碳酸 钾为缚酸剂, 乙腈为溶剂, 加热回流的方法顺利地制备 出了其它含苯并三氮唑结构的吡唑肜衍生物.

\section{2 目标产物 7 的谱图剖析}

以目标产物 7e 为例, 对 $7 \mathrm{e}$ 的 ${ }^{1} \mathrm{H}$ NMR 和 ${ }^{13} \mathrm{C} \mathrm{NMR}$ 数据进行剖析. 由 $7 \mathrm{e}$ 的核磁氢谱可看出, $\delta 8.16$ 出现的

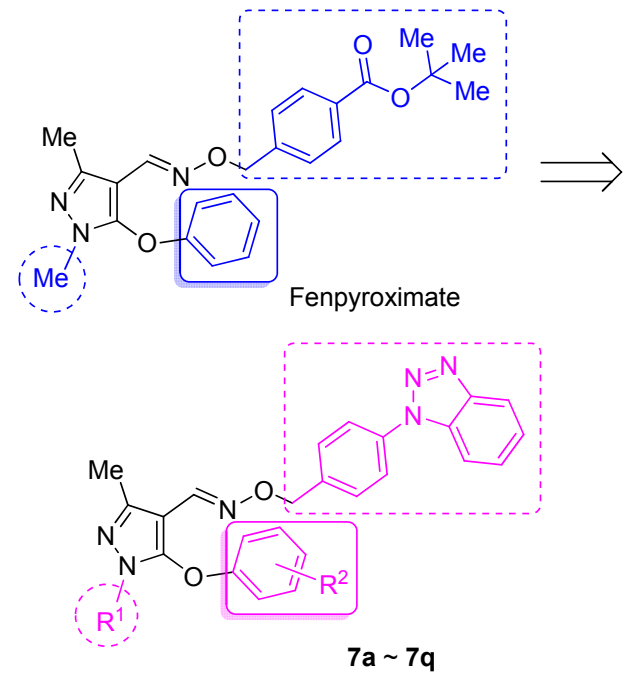

图 2 目标化合物 7a $\sim \mathbf{q}$ 的分子设计图

Figure 2 Design strategy of the title compounds $7 \mathbf{a} \sim 7 \mathbf{q}$

表 1 反应条件对化合物 7g 收率的影响

Table 1 Effects of reaction conditions on the synthesis of compound $7 \mathrm{~g}$

\begin{tabular}{cllcc}
\hline Entry & Base & Solvent & Reaction condition & Yield/\% \\
\hline 1 & $\mathrm{Et}_{3} \mathrm{~N}$ & $\mathrm{CHCl}_{3}$ & Reflux for $10 \mathrm{~h}$ & 0 \\
2 & $\mathrm{Na}_{2} \mathrm{CO}_{3}$ & $\mathrm{CHCl}_{3}$ & Reflux for $10 \mathrm{~h}$ & 0 \\
3 & $\mathrm{~K}_{2} \mathrm{CO}_{3}$ & $\mathrm{CHCl}_{3}$ & Reflux for $10 \mathrm{~h}$ & 0 \\
4 & $\mathrm{Et}_{3} \mathrm{~N}$ & $\mathrm{CH}_{3} \mathrm{CN}$ & Reflux for $10 \mathrm{~h}$ & 0 \\
5 & $\mathrm{Na}_{2} \mathrm{CO}_{3}$ & $\mathrm{CH}_{3} \mathrm{CN}$ & Reflux for $10 \mathrm{~h}$ & 0 \\
6 & $\mathrm{~K}_{2} \mathrm{CO}_{3}$ & $\mathrm{CH}_{3} \mathrm{CN}$ & Reflux for $10 \mathrm{~h}$ & 70 \\
7 & $\mathrm{KOH}$ & $\mathrm{CH}_{3} \mathrm{CN}$ & Reflux for $10 \mathrm{~h}$ & 32 \\
8 & $\mathrm{Et}_{3} \mathrm{~N}$ & $\mathrm{DMF}$ & $80{ }^{\circ} \mathrm{C}$ for $10 \mathrm{~h}$ & 0 \\
9 & $\mathrm{Na}_{2} \mathrm{CO}_{3}$ & $\mathrm{DMF}$ & $80{ }^{\circ} \mathrm{C}$ for $10 \mathrm{~h}$ & 0 \\
10 & $\mathrm{~K}_{2} \mathrm{CO}_{3}$ & $\mathrm{DMF}$ & $80{ }^{\circ} \mathrm{C}$ for $10 \mathrm{~h}$ & 55 \\
11 & $\mathrm{KOH}^{\circ}$ & $\mathrm{DMF}$ & $80{ }^{\circ} \mathrm{C}$ for $10 \mathrm{~h}$ & 0 \\
\hline
\end{tabular}

双重峰归属于苯并三氮唑苯环上的 1 个氢; $\delta 7.87$ 出现 的单峰归属于 $\mathrm{CH}=\mathrm{N}$ 键上的 1 个氢; $\delta 7.73 \sim 7.76$ 出现 的多重峰归属于苯并三氮唑苯环上的 1 个氢及与苯并三 氮唑相连的苯环上的 2 个氢; $\delta 7.56 \sim 7.58$ 出现的多重峰 归属于苯并三氮唑苯环上的 1 个氢; $\delta 7.51$ 出现的双重 峰归属于与苯并三氮唑相连的苯环上的另外 2 个氢; $\delta$ 7.43 7.47 出现的多重峰归属于苯并三氮唑苯环上的另 外 1 个氢; $\delta 6.81 \sim 7.24$ 出现的多重峰归属于 $3-\mathrm{Br}$ 取代 苯环上的 4 个氢; $\delta 5.08$ 出现的单峰归属于与氧原子相 连的 $\mathrm{CH}_{2}$ 上的 2 个氢; $\delta 3.62$ 出现的单峰归属于取代吡 唑环 1-位 $\mathrm{CH}_{3}$ 上的 3 个氢; $\delta 2.38$ 出现的单峰归属于取 代吡唑环 3-位 $\mathrm{CH}_{3}$ 上的 3 个氢. 从 $7 \mathrm{e}$ 的核磁碳谱可知, $\mathrm{OCH}_{2}$ 的 $\mathrm{C}$ 原子出现在 $\delta 75.24$, 取代吡唑环 1-位 $\mathrm{CH}_{3}$ 和 3 -位 $\mathrm{CH}_{3}$ 的 $\mathrm{C}$ 原子分别出现在 $\delta 34.30$ 和 14.47 .

\section{3 生物活性}

采用喷雾法 ${ }^{[27]}$ 测定目标化合物 $7 \mathbf{a} \sim \mathbf{7 q}$ 的杀朱砂叶 

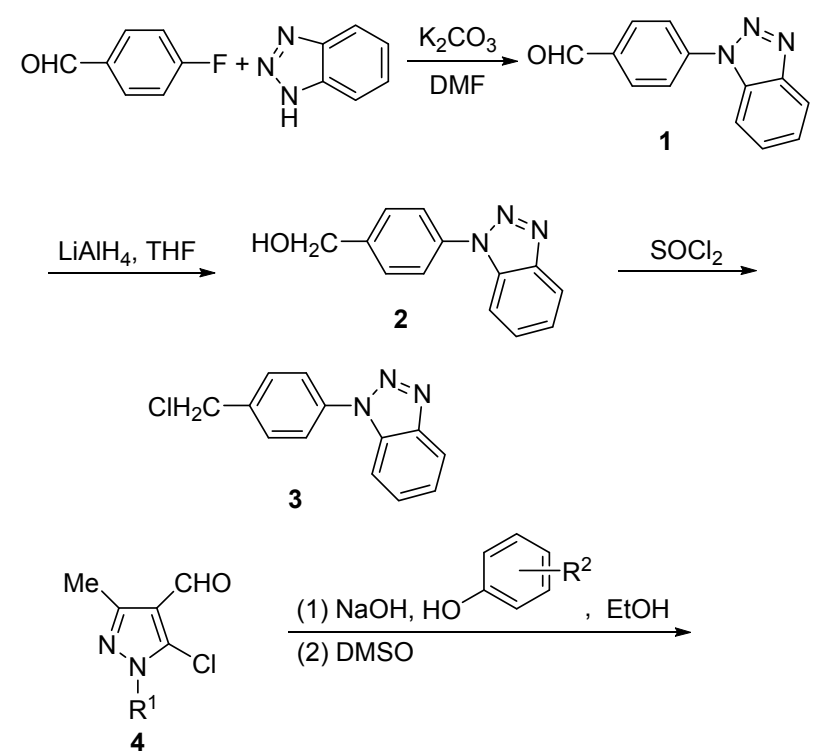

(2) DMSO

4
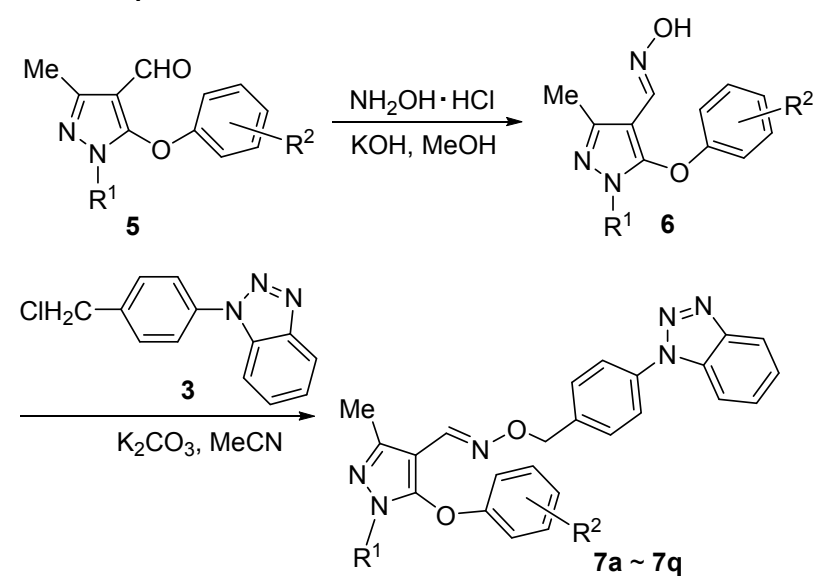

7a: $R^{1}=M e, R^{2}=3-F ; 7 b: R^{1}=M e, R^{2}=4-F ; 7 c: R^{1}=M e, R^{2}=3-C l ;$ 7d: $R^{1}=M e, R^{2}=4-C l ; 7 e: R^{1}=M e, R^{2}=3-B r ; 7 f: R^{1}=M e, R^{2}=$ 4-Br; 7g: $\mathrm{R}^{1}=\mathrm{Me}, \mathrm{R}^{2}=\mathrm{H} ; \mathbf{7 h}: \mathrm{R}^{1}=\mathrm{Me}, \mathrm{R}^{2}=2$-OMe; $7 \mathrm{i}: \mathrm{R}^{1}=\mathrm{Me}$, $\mathrm{R}^{2}=4$-OMe; 7j: $\mathrm{R}^{1}=\mathrm{Me}, \mathrm{R}^{2}=4-\mathrm{OCF}_{3} ; 7 \mathrm{k}: \mathrm{R}^{1}=\mathrm{Me}, \mathrm{R}^{2}=3-\mathrm{Me}$; 7l: $R^{1}=M e, R^{2}=4-M e ; 7 m: R^{1}=M e, R^{2}=4-C_{3} ; 7 n: R^{1}=M e$, $\mathrm{R}^{2}=2,3-\mathrm{F}_{2} ; 7 \mathrm{o}: \mathrm{R}^{1}=\mathrm{Me}, \mathrm{R}^{2}=2,4-\mathrm{Cl}_{2} ; 7 \mathrm{p}: \mathrm{R}^{1}=4-\mathrm{MeC}_{6} \mathrm{H}_{4}, \mathrm{R}^{2}=4-\mathrm{F}$; $7 q: \mathrm{R}^{1}=4-\mathrm{MeC}_{6} \mathrm{H}_{4}, \mathrm{R}^{2}=4-\mathrm{Me}$

图式 1 目标化合物 $7 \mathbf{a} \sim 7 \mathbf{q}$ 的合成路线

Scheme 1 Synthetic route of the target compounds $7 \mathbf{a} \sim 7 \mathbf{q}$

螨 (Tetranychus cinnabarinus) 及杀蚜虫 (Aphis medicaginis)活性, 利用浸叶法 ${ }^{[28]}$ 测定目标化合物 $7 \mathbf{a} \sim 7 \mathbf{q}$ 的 杀粘虫(Mythimna separata)活性. 通过表 2 可以看出, 部 分目标化合物对所测试的三种昆虫呈现出良好的杀虫 效果. 在 $500 \mu \mathrm{g} / \mathrm{mL}$ 测试浓度下, 7a、7b、7d、7f、7g、 $7 \mathbf{i} 、 7 \mathbf{j}$ 和 71 对朱砂叶螨有 $100 \%$ 的致死率，与唑螨酯相 当; 一些化合物在 $100 \mu \mathrm{g} / \mathrm{mL}$ 浓度下对朱砂叶螨有明显 的杀虫效果, 其中 $7 \mathbf{a} 、 7 \mathbf{b} 、 7 \mathbf{f} 、 7 \mathbf{i}$ 和 $7 \mathbf{j}$ 对朱砂叶螨的杀 死率为 $90 \% \sim 100 \%$, 与唑螨酯相接近; 测试浓度降到 $20 \mu \mathrm{g} / \mathrm{mL}$ 时，化合物 7a、7b、7f、7i 和 7j 对朱砂叶螨 仍表现出 $60 \% \sim 100 \%$ 的杀灭效果, 其中 $7 \mathbf{a}$ 和 $7 \mathbf{j}$ 与唑螨 酯的杀虫活性相当. 通过构效关系分析能看出, 当 $\mathrm{R}^{1}=$
$\mathrm{CH}_{3}$ 时, $\mathrm{R}^{2}$ 为 3-F (7a)、4-F (7b)、4-Br (7f)、4-OCH $3(\mathbf{7} \mathbf{i})$ 、 $4-\mathrm{OCF}_{3}(7 \mathbf{j})$ 的化合物对朱砂叶螨的防效要高于其它化合 物的杀虫活性. 同时, 多数目标化合物在 $500 \mu \mathrm{g} / \mathrm{mL}$ 浓 度下对蚛虫有着较高的杀虫活性，其中 $7 \mathbf{a} \sim 7 \mathrm{~g}$ 和 $7 \mathbf{i} \sim 7 \mathbf{7}$ 对蚜虫的杀死率为 $100 \%$, 与对照药吡虫啉相当; 化合 物 7a $\sim 7 \mathbf{d} 、 7 \mathbf{f} \sim 7 \mathrm{~g}$ 和 $7 \mathbf{i} \sim 7 \mathbf{1}$ 在 $100 \mu \mathrm{g} / \mathrm{mL}$ 浓度下对蚜 虫还有 $100 \%$ 的杀虫作用; 降低测试浓度至 $20 \mu \mathrm{g} / \mathrm{mL}$, 一些目标化合物对蚜虫仍表现出较好的杀灭作用，化合 物 7a、7b、7d、7f、7 $\mathrm{g}$ 和 7i $\sim 71$ 对蚜虫的致死率为 $70 \%$ $100 \%$. 从构效关系分析发现，当 $\mathrm{R}^{1}=\mathrm{CH}_{3}$ 时, $\mathrm{R}^{2}$ 为 $3-\mathrm{F}$ (7a)、4-F (7b)、4-Br (7f) 、4- $\mathrm{OCH}_{3}(\mathbf{7 i}) 、 4-\mathrm{OCF}_{3}(\mathbf{7 j}) 、 4-\mathrm{CH}_{3}$ (71) 的化合物对蚜虫的杀虫活性较高, 要好于其它目标 化合物对蚜虫的防效. 在 $4 \mu \mathrm{g} / \mathrm{mL}$ 浓度下，化合物 7a、

7f、7i 和 7k 对蚜虫仍有 $20 \% \sim 30 \%$ 的杀死率. 此外，在 $500 \mu \mathrm{g} / \mathrm{mL}$ 浓度下, 多数化合物对粘虫也有较高的防治 作用，其中化合物 7a 7o 对粘虫的致死率都达 100\%, 与对照药啶虫丙醚相当. 通过构效关系分析可知, $\mathrm{R}^{1}=$ $\mathrm{CH}_{3}$ 时化合物 $7 \mathbf{a} \sim 7 \mathbf{0}$ 对粘虫的杀虫活性要高于 $\mathrm{R}^{1}=$ 4- $\mathrm{CH}_{3} \mathrm{C}_{6} \mathrm{H}_{4}$ 时化合物 7p 和 $7 \mathbf{q}$ 的防效. 从整体的构效关 系也能看出, 化合物 7a $\left(\mathrm{R}^{1}=\mathrm{CH}_{3}, \mathrm{R}^{2}=3-\mathrm{F}\right) 、 \mathbf{7 f}\left(\mathrm{R}^{1}=\right.$ $\left.\mathrm{CH}_{3}, \mathrm{R}^{2}=4-\mathrm{Br}\right)$ 和 $7 \mathbf{j}\left(\mathrm{R}^{1}=\mathrm{CH}_{3}, \mathrm{R}^{2}=4-\mathrm{OCF}_{3}\right)$ 对所测试的 朱砂叶螨、蚜虫和粘虫的杀虫效果相对突出, 可作进一 步的结构衍生与活性研究.

\section{2 结论}

通过活性单元拼接方法, 合成出 17 个具有苯并三 氮唑单元的吡唑肟衍生物. 通过核磁共振氢谱、核磁碳 谱及元素分析对目标化合物的结构进行了确认. 初步杀 虫活性测试数据显示, 在 $500 \mu \mathrm{g} / \mathrm{mL}$ 测试浓度下, 目标 化合物 7a、7b、7d、7f、7g、7i、7j 和 71 对朱砂叶螨 的杀死率均达 $100 \%$, 测试浓度降至 $100 \mu \mathrm{g} / \mathrm{mL}$ 时, 目标 化合物 7a、7b、7f、7i 和 7j 对朱砂叶螨表现出 $90 \%$ $100 \%$ 的杀虫作用, 7a、7f 和 7 $\mathbf{j}$ 在 $20 \mu \mathrm{g} / \mathrm{mL}$ 浓度下对朱 砂叶螨仍有较高的杀灭效果，杀死率分别 $90 \%, 70 \%$ 和 $100 \%$; 化合物 $7 \mathbf{a} \sim 7 \mathbf{d} 、 7 \mathbf{f} \sim 7 \mathbf{g}$ 和 $7 \mathbf{i} \sim 71$ 在 500 和 100 $\mu \mathrm{g} / \mathrm{mL}$ 浓度下对蚜虫有优异的防效，致死率均为 $100 \%$, 降低测试浓度至 $20 \mu \mathrm{g} / \mathrm{mL}$ 时，化合物 7a、7b、7f、7i、 $7 \mathbf{j}$ 和 71 对蚜虫仍有 $90 \%$ 以上的杀虫活性. 此外, 化合物 $7 \mathbf{a} \sim 7 \mathbf{p}$ 在 $500 \mu \mathrm{g} / \mathrm{mL}$ 浓度下对粘虫具有 $80 \% \sim 100 \%$ 的 防效. 这为今后继续进行新型吡唑肜分子合成和杀虫活 性研究提供了重要的参考信息.

\section{3 实验部分}

\section{1 仪器与试剂}

通过 BRUKER $400 \mathrm{MHz}$ 核磁共振仪(TMS 为内标) 
表 2 目标化合物 $7 \mathbf{a} \sim 7 \mathbf{q}$ 的杀虫活性(死亡率 $/ \%)^{a}$

Table 2 Insecticidal activities of the title compounds $\mathbf{7 a} \sim \mathbf{7 q}$ (mortality/\%)

\begin{tabular}{|c|c|c|c|c|c|c|c|c|c|c|}
\hline \multirow{2}{*}{ Compd. } & \multicolumn{4}{|c|}{ Tetranychus cinnabarinus } & \multicolumn{4}{|c|}{ Aphis medicaginis } & \multicolumn{2}{|c|}{ Mythimna separata } \\
\hline & $500 \mu \mathrm{g} / \mathrm{mL}$ & $100 \mu \mathrm{g} / \mathrm{mL}$ & $20 \mu \mathrm{g} / \mathrm{mL}$ & $4 \mu \mathrm{g} / \mathrm{mL}$ & $500 \mu \mathrm{g} / \mathrm{mL}$ & $100 \mu \mathrm{g} / \mathrm{mL}$ & $20 \mu \mathrm{g} / \mathrm{mL}$ & $4 \mu \mathrm{g} / \mathrm{mL}$ & $500 \mu \mathrm{g} / \mathrm{mL}$ & $100 \mu \mathrm{g} / \mathrm{mL}$ \\
\hline $7 a$ & 100 & 100 & 90 & 0 & 100 & 100 & 100 & 30 & 100 & 0 \\
\hline $7 b$ & 100 & 100 & 60 & 0 & 100 & 100 & 90 & 0 & 100 & 0 \\
\hline $7 \mathrm{c}$ & 0 & - & - & - & 100 & 100 & 50 & 0 & 100 & 0 \\
\hline $7 d$ & 100 & 0 & - & - & 100 & 100 & 70 & 0 & 100 & 0 \\
\hline $7 e$ & 0 & - & - & - & 100 & 70 & 20 & 0 & 100 & 0 \\
\hline $7 f$ & 100 & 100 & 70 & 0 & 100 & 100 & 100 & 30 & 100 & 0 \\
\hline $7 \mathrm{~g}$ & 100 & 0 & - & - & 100 & 100 & 70 & 0 & 100 & 0 \\
\hline $7 \mathrm{~h}$ & 0 & - & - & - & 0 & - & - & - & 100 & 0 \\
\hline $7 \mathbf{i}$ & 100 & 90 & 60 & 0 & 100 & 100 & 100 & 20 & 100 & 0 \\
\hline $7 \mathbf{j}$ & 100 & 100 & 100 & 0 & 100 & 100 & 90 & 0 & 100 & 0 \\
\hline $7 \mathbf{k}$ & 0 & - & - & - & 100 & 100 & 80 & 30 & 100 & 0 \\
\hline 71 & 100 & 70 & 0 & - & 100 & 100 & 90 & 0 & 100 & 0 \\
\hline $7 \mathrm{~m}$ & 0 & - & - & - & 0 & - & - & - & 100 & 0 \\
\hline $7 n$ & 0 & - & - & - & 0 & - & - & - & 100 & 0 \\
\hline 70 & 0 & - & - & - & 60 & 0 & - & - & 100 & 0 \\
\hline $7 p$ & 0 & - & - & - & 0 & - & - & - & 80 & 0 \\
\hline $7 q$ & 0 & - & - & - & 0 & - & - & - & 0 & - \\
\hline Fenpyroximate & 100 & 100 & 100 & - & - & - & - & - & - & - \\
\hline Imidacloprid & - & - & - & - & 100 & 100 & 100 & 100 & - & - \\
\hline Pyridalyl & - & - & - & - & - & - & - & - & 100 & 100 \\
\hline
\end{tabular}

a - refers to "not tested".

测定化合物的核磁氢谱和碳谱; 通过 X-4 显微熔点测定 仪(温度计未经校正)测定化合物的熔点; 通过 YanacoCHN CORDER MT-3 自动元素分析仪测试化合物的元 素分析. 所用的试剂都为分析纯. 化合物 $\mathbf{1}^{[29]}$ 和 $4 \sim \mathbf{6}^{[30]}$ 参照文献合成.

\section{2 化合物 2 的合成}

将 $0.02 \mathrm{~mol}$ 化合物 $\mathbf{1} 、 80 \mathrm{~mL}$ 四氢呋喃加入 $250 \mathrm{~mL}$ 反应瓶中, 冰浴条件下向其中分批加入 $0.06 \mathrm{~mol}$ 氢化铝 锂, 加好后, 冰浴搅拌 $2 \mathrm{~h}$. 反应结束后, 向混合液中加 入 $30 \mathrm{~mL}$ 水, 过滤, 用 $50 \mathrm{~mL}$ 二氯甲烷洗涤固体, 母液 静置分层, 水相用二氯甲烷萃取 $(30 \mathrm{~mL} \times 3)$, 合并后的 有机相通过无水硫酸钠干燥、脱溶得到化合物 $\mathbf{2}$, 不经 纯化可直接投于下步反应.

\section{3 化合物 3 的合成}

将 $0.01 \mathrm{~mol}$ 化合物 2、50 mL 二氯甲烷加入 $100 \mathrm{~mL}$ 反应瓶中, 冰浴条件下向其中缓慢滴加 $0.02 \mathrm{~mol}$ 二氯亚 砜, 加好后, 室温搅拌 $3 \mathrm{~h}$. 反应结束后, 向混合液中加 入 $30 \mathrm{~mL}$ 水, 用饱和碳酸氢钠溶液调节 $\mathrm{pH}$ 至 7 , 静置分 层, 有机相经无水硫酸钠干燥、脱溶得到粗品, 通过柱 层析 $[V($ 乙酸乙酯 $): V($ 石油醚 $)=1: 20]$ 分离得白色固 体, 产率 77\%. m.p. $112 \sim 113{ }^{\circ} \mathrm{C} ;{ }^{1} \mathrm{H}$ NMR $\left(\mathrm{CDCl}_{3}, 400\right.$ $\mathrm{MHz}) \delta: 8.16(\mathrm{~d}, J=8.00 \mathrm{~Hz}, 1 \mathrm{H}), 7.81$ (d, $J=8.40 \mathrm{~Hz}$, 2H), 7.76 (d, $J=8.40 \mathrm{~Hz}, 1 \mathrm{H}), 7.65$ (d, $J=8.40 \mathrm{~Hz}, 2 \mathrm{H})$, $7.55 \sim 7.59(\mathrm{~m}, 1 \mathrm{H}), 7.43 \sim 7.47(\mathrm{~m}, 1 \mathrm{H}), 4.69(\mathrm{~s}, 2 \mathrm{H})$.
Anal. calcd for $\mathrm{C}_{13} \mathrm{H}_{10} \mathrm{ClN}_{3}$ : C 64.07, $\mathrm{H}$ 4.14, $\mathrm{N}$ 17.24; found $\mathrm{C} 64.25, \mathrm{H} 4.30, \mathrm{~N} 17.12$.

\section{4 目标化合物 $7 a \sim 7 q$ 的合成}

将 $1 \mathrm{mmol}$ 化合物 6、1.2 mmol 化合物 3 及 $30 \mathrm{~mL}$ 乙腈加到 $50 \mathrm{~mL}$ 反应瓶中, 室温搅拌下向其中加入 2 $\mathrm{mmol}$ 碳酸钾. 加好后, 将反应液升温回流反应 $8 \sim 16 \mathrm{~h}$. 停止反应, 冷却至室温, 抽滤, 用 $20 \mathrm{~mL}$ 乙腈洗涤固体, 接下来浓缩母液所得粗品通过柱层析 $[V($ 乙酸乙酯 $)$ : $V($ 石油醚 $)=1 ： 15]$ 分离得到目标产物 $7 \mathbf{a} \sim 7 \mathbf{q}$.

$O$-[4-(1H-苯并三唑-1-基)芐基]-5-(3-氟苯氧基)-3-甲 基-1-甲基- $1 H$-吡唑-4-甲酰基肟(7a)：白色固体，产率 61\%. m.p. 98 $100{ }^{\circ} \mathrm{C} ;{ }^{1} \mathrm{H}$ NMR $\left(\mathrm{CDCl}_{3}, 400 \mathrm{MHz}\right) \delta$ : $8.16(\mathrm{~d}, J=8.40 \mathrm{~Hz}, 1 \mathrm{H}), 7.87(\mathrm{~s}, 1 \mathrm{H}), 7.73 \sim 7.75(\mathrm{~m}$, $3 \mathrm{H}), 7.54 \sim 7.58(\mathrm{~m}, 1 \mathrm{H}), 7.52(\mathrm{~d}, J=8.40 \mathrm{~Hz}, 2 \mathrm{H}), 7.43 \sim$ $7.47(\mathrm{~m}, 1 \mathrm{H}), 7.23 \sim 7.27(\mathrm{~m}, 1 \mathrm{H}), 6.78 \sim 6.83(\mathrm{~m}, 1 \mathrm{H})$, $6.62 \sim 6.69(\mathrm{~m}, 2 \mathrm{H}), 5.09(\mathrm{~s}, 2 \mathrm{H}), 3.61(\mathrm{~s}, 3 \mathrm{H}), 2.38(\mathrm{~s}$, $3 \mathrm{H}) ;{ }^{13} \mathrm{C} \mathrm{NMR}\left(\mathrm{CDCl}_{3}, 100 \mathrm{MHz}\right) \delta: 163.51(\mathrm{~d}, J=246.50$ $\mathrm{Hz})$, 157.69, 157.58, 147.06, 146.53, 140.81, 138.48, $136.46,132.29$, 130.96, 129.79, 128.28, 124.43, 122.71, 120.38, 110.94, 110.49 (d, $J=24.00 \mathrm{~Hz}), 103.54$ (d, $J=$ $25.80 \mathrm{~Hz}), 100.30,75.23,34.28,14.54$. Anal. calcd for $\mathrm{C}_{25} \mathrm{H}_{21} \mathrm{FN}_{6} \mathrm{O}_{2}$ : C 65.78, H 4.64, N 18.41; found $\mathrm{C} 65.95, \mathrm{H}$ 4.51, N 18.61 .

$O$-[4-(1H-苯并三唑-1-基)芐基]-5-(4-氟苯氧基)-3-甲 
基-1-甲基-1H-吡唑-4-甲酰基肜(7b)：无色油状物，产率 66\%. ${ }^{1} \mathrm{H}$ NMR $\left(\mathrm{CDCl}_{3}, 400 \mathrm{MHz}\right) \delta: 8.16(\mathrm{~d}, J=8.40 \mathrm{~Hz}$, $1 \mathrm{H}), 7.85(\mathrm{~s}, 1 \mathrm{H}), 7.75(\mathrm{~d}, J=8.40 \mathrm{~Hz}, 3 \mathrm{H}), 7.43 \sim 7.58$ $(\mathrm{m}, 4 \mathrm{H}), 6.98 \sim 7.02(\mathrm{~m}, 2 \mathrm{H}), 6.84 \sim 6.88(\mathrm{~m}, 2 \mathrm{H}), 5.09(\mathrm{~s}$, 2H), $3.61(\mathrm{~s}, 3 \mathrm{H}), 2.37(\mathrm{~s}, 3 \mathrm{H}) ;{ }^{13} \mathrm{C} \mathrm{NMR}\left(\mathrm{CDCl}_{3}, 100\right.$ $\mathrm{MHz}) \delta: 158.77$ (d, $J=241.10 \mathrm{~Hz}), 152.63,147.76$, 147.02 , 146.54, 140.91, 138.47, 136.49, 132.28, 129.81, $129.37,128.29,124.43,122.70,120.38,116.53$ (d, $J=$ 23.50 Hz), 110.35, 99.99, 75.22, 34.25, 14.63. Anal. calcd for $\mathrm{C}_{25} \mathrm{H}_{21} \mathrm{FN}_{6} \mathrm{O}_{2}$ : C 65.78, $\mathrm{H}$ 4.64, $\mathrm{N} \mathrm{18.41;} \mathrm{found} \mathrm{C}$ 65.62, H 4.49, N 18.53.

$O$-[4-(1H-苯并三唑-1-基)芐基]-5-(3-氯苯氧基)-3-甲 基-1-甲基- $1 H$-吡唑-4-甲酰基肟(7c): 白色固体, 产率 65\%. m.p. 137 139 ${ }^{\circ} \mathrm{C} ;{ }^{1} \mathrm{H}$ NMR $\left(\mathrm{CDCl}_{3}, 400 \mathrm{MHz}\right) \delta$ : $8.16(\mathrm{~d}, J=8.40 \mathrm{~Hz}, 1 \mathrm{H}), 7.87(\mathrm{~s}, 1 \mathrm{H}), 7.73 \sim 7.76(\mathrm{~m}$, $3 \mathrm{H}), 7.54 \sim 7.58(\mathrm{~m}, 1 \mathrm{H}), 7.51(\mathrm{~d}, J=8.40 \mathrm{~Hz}, 2 \mathrm{H}), 7.43 \sim$ $7.47(\mathrm{~m}, 1 \mathrm{H}), 6.77 \sim 7.25(\mathrm{~m}, 4 \mathrm{H}), 5.08(\mathrm{~s}, 2 \mathrm{H}), 3.61(\mathrm{~s}$, $3 \mathrm{H}), 2.37(\mathrm{~s}, 3 \mathrm{H}) ;{ }^{13} \mathrm{C} \mathrm{NMR}\left(\mathrm{CDCl}_{3}, 100 \mathrm{MHz}\right) \delta: 157.15$, $147.12,146.83,146.52,140.78,138.48,136.44,135.44$, $132.28,130.79,129.78,128.29,124.44,123.97,122.70$, $120.36,115.93,113.56,110.38,100.27,75.23,34.29$, 14.49. Anal. calcd for $\mathrm{C}_{25} \mathrm{H}_{21} \mathrm{ClN}_{6} \mathrm{O}_{2}$ : C 63.49, $\mathrm{H} 4.48, \mathrm{~N}$ 17.77; found $\mathrm{C} 63.30, \mathrm{H} 4.65, \mathrm{~N} 17.90$.

$O$-[4-(1H-苯并三唑-1-基)芐基]-5-(4-氯苯氧基)-3-甲 基-1-甲基- $1 H$-吡唑-4-甲酰基肟(7d)：白色固体，产率 68\%. m.p. $101 \sim 103{ }^{\circ} \mathrm{C} ;{ }^{1} \mathrm{H}$ NMR $\left(\mathrm{CDCl}_{3}, 400 \mathrm{MHz}\right) \delta$ : $8.15(\mathrm{~d}, J=8.00 \mathrm{~Hz}, 1 \mathrm{H}), 7.85$ (s, 1H), 7.75 (d, $J=8.00$ $\mathrm{Hz}, 3 \mathrm{H}), 7.43 \sim 7.58(\mathrm{~m}, 4 \mathrm{H}), 7.25 \sim 7.28(\mathrm{~m}, 2 \mathrm{H}), 6.84(\mathrm{~d}$, $J=8.80 \mathrm{~Hz}, 2 \mathrm{H}), 5.08$ (s, 2H), 3.62 (s, 3H), 2.37 (s, 3H); ${ }^{13} \mathrm{C} \mathrm{NMR}\left(\mathrm{CDCl}_{3}, 100 \mathrm{MHz}\right) \delta: 155.22,147.19,147.07$, $146.54,140.80,138.44,136.49,132.28,129.94,129.82$, $128.77,128.29,124.43,122.69,120.38,116.63,110.37$, $100.15,75.25,34.26,14.52$. Anal. calcd for $\mathrm{C}_{25} \mathrm{H}_{21} \mathrm{ClN}_{6} \mathrm{O}_{2}$ : C 63.49, H 4.48, N 17.77; found C 63.65, H 4.59, N 17.62.

$O$-[4-(1H-苯并三唑-1-基)苄基]-5-(3-溴苯氧基)-3-甲 基-1-甲基- $1 H$-吡唑-4-甲酰基肜(7e)：黄色固体，产率 63\%. m.p. $143 \sim 145{ }^{\circ} \mathrm{C} ;{ }^{1} \mathrm{H}$ NMR $\left(\mathrm{CDCl}_{3}, 400 \mathrm{MHz}\right) \delta$ : $8.16(\mathrm{~d}, J=8.40 \mathrm{~Hz}, 1 \mathrm{H}), 7.87(\mathrm{~s}, 1 \mathrm{H}), 7.73 \sim 7.76(\mathrm{~m}$, $3 \mathrm{H}), 7.56 \sim 7.58(\mathrm{~m}, 1 \mathrm{H}), 7.51(\mathrm{~d}, J=8.40 \mathrm{~Hz}, 2 \mathrm{H}), 7.43 \sim$ $7.47(\mathrm{~m}, 1 \mathrm{H}), 6.81 \sim 7.24(\mathrm{~m}, 4 \mathrm{H}), 5.08(\mathrm{~s}, 2 \mathrm{H}), 3.62(\mathrm{~s}$, $3 \mathrm{H}), 2.38(\mathrm{~s}, 3 \mathrm{H}) ;{ }^{13} \mathrm{C} \mathrm{NMR}\left(\mathrm{CDCl}_{3}, 100 \mathrm{MHz}\right) \delta: 157.16$, $147.13,146.79,146.53,140.77,138.49,136.45,132.29$, $131.09,129.78,128.28,126.88,124.43,123.17,122.71$, $120.37,118.79$, 114.03, 110.39, 100.26, 75.24, 34.30, 14.47. Anal. calcd for $\mathrm{C}_{25} \mathrm{H}_{21} \mathrm{BrN}_{6} \mathrm{O}_{2}$ : C 58.04, $\mathrm{H} 4.09, \mathrm{~N}$
16.24; found C 58.24, H 4.01, N 16.06.

$O$-[4-(1H-苯并三唑-1-基)芐基]-5-(4-溴苯氧基)-3-甲 基-1-甲基- $1 H$-吡唑-4-甲酰基肟(7f)：黄色固体，产率 67\%. m.p. 85 87 ${ }^{\circ} \mathrm{C} ;{ }^{1} \mathrm{H}$ NMR $\left(\mathrm{CDCl}_{3}, 400 \mathrm{MHz}\right) \delta$ : $8.16(\mathrm{~d}, J=8.00 \mathrm{~Hz}, 1 \mathrm{H}), 7.85$ (s, 1H), 7.75 (d, $J=8.00$ $\mathrm{Hz}, 3 \mathrm{H}), 7.49 \sim 7.58(\mathrm{~m}, 3 \mathrm{H}), 7.40 \sim 7.47(\mathrm{~m}, 3 \mathrm{H}), 6.78(\mathrm{~d}$, $J=8.80 \mathrm{~Hz}, 2 \mathrm{H}), 5.08$ (s, 2H), 3.61 (s, 3H), 2.37 (s, 3H); ${ }^{13} \mathrm{C} \mathrm{NMR}\left(\mathrm{CDCl}_{3}, 100 \mathrm{MHz}\right) \delta: 154.70,146.02,145.49$, $139.70,137.38,135.44,131.85,131.23,128.77,128.34$, $127.24,123.38,121.65,119.33,116.03,115.12,109.33$, 99.14, 74.21, 33.21, 13.43. Anal. calcd for $\mathrm{C}_{25} \mathrm{H}_{21} \mathrm{BrN}_{6} \mathrm{O}_{2}$ : C 58.04, H 4.09, N 16.24; found C 57.87, H 4.23, N 16.43.

$O$-[4-(1H-苯并三唑-1-基)苄基]-5-苯氧基-3-甲基-1甲基- $1 H$-吡唑-4-甲酰基肜(7g)：白色固体，产率 70\%. m.p. 89 91 ${ }^{\circ} \mathrm{C} ;{ }^{1} \mathrm{H}$ NMR $\left(\mathrm{CDCl}_{3}, 400 \mathrm{MHz}\right) \delta: 8.15(\mathrm{~d}$, $J=8.40 \mathrm{~Hz}, 1 \mathrm{H}), 7.86(\mathrm{~s}, 1 \mathrm{H}), 7.72 \sim 7.75(\mathrm{~m}, 3 \mathrm{H}), 7.51 \sim$ $7.58(\mathrm{~m}, 3 \mathrm{H}), 7.43 \sim 7.46(\mathrm{~m}, 1 \mathrm{H}), 7.30 \sim 7.34(\mathrm{~m}, 2 \mathrm{H})$, $7.08 \sim 7.12(\mathrm{~m}, 1 \mathrm{H}), 6.90(\mathrm{~d}, J=8.00 \mathrm{~Hz}, 2 \mathrm{H}), 5.10(\mathrm{~s}$, $2 \mathrm{H}), 3.61(\mathrm{~s}, 3 \mathrm{H}), 2.38(\mathrm{~s}, 3 \mathrm{H}) ;{ }^{13} \mathrm{C} \mathrm{NMR}\left(\mathrm{CDCl}_{3}, 100\right.$ $\mathrm{MHz}) \delta$ : 156.72, 147.76, 146.93, 146.53, 141.16, 138.54, $136.43,132.29,130.03,129.88,128.27,124.42,123.71$, $122.71,120.37,115.29,110.37,100.18,75.20,34.26$, 14.77. Anal. calcd for $\mathrm{C}_{25} \mathrm{H}_{22} \mathrm{~N}_{6} \mathrm{O}_{2}$ : C 68.48, H 5.06, N 19.17; found C 68.36, H 5.19, N 19.01.

$O$-[4-(1H-苯并三唑-1-基)苄基]-5-(2-甲氧基苯氧 基)-3-甲基-1-甲基- $1 H$-吡唑-4-甲酰基肟(7h): 黄色固体, 产率 65\%. m.p. $73 \sim 75{ }^{\circ} \mathrm{C} ;{ }^{1} \mathrm{H} \mathrm{NMR}\left(\mathrm{CDCl}_{3}, 400 \mathrm{MHz}\right)$ $\delta: 8.15(\mathrm{~d}, J=8.00 \mathrm{~Hz}, 1 \mathrm{H}), 7.83(\mathrm{~s}, 1 \mathrm{H}), 7.73(\mathrm{~d}, J=8.40$ $\mathrm{Hz}, 3 \mathrm{H}), 7.42 \sim 7.57(\mathrm{~m}, 4 \mathrm{H}), 6.68 \sim 7.10(\mathrm{~m}, 4 \mathrm{H}), 5.10(\mathrm{~s}$, 2H), $3.91(\mathrm{~s}, 3 \mathrm{H}), 3.63(\mathrm{~s}, 3 \mathrm{H}), 2.37(\mathrm{~s}, 3 \mathrm{H}) ;{ }^{13} \mathrm{C} \mathrm{NMR}$ $\left(\mathrm{CDCl}_{3}, 100 \mathrm{MHz}\right) \delta: 148.97,148.47,146.80,146.52$, $145.76,141.27,138.66,136.40,132.30,129.79$, 128.27, $124.61,124.42,122.72,120.96,120.36,115.80,112.77$, $110.37,99.58,75.11,56.09,34.22,14.91$. Anal. calcd for $\mathrm{C}_{26} \mathrm{H}_{24} \mathrm{~N}_{6} \mathrm{O}_{3}$ : C 66.65, H 5.16, N 17.94; found C 66.48, H $5.01, \mathrm{~N} 18.12$.

$O$-[4-(1H-苯并三唑-1-基)苄基]-5-(4-甲氧基苯氧 基)-3-甲基-1-甲基- $1 H$-吡唑-4-甲酰基肟(7i): 黄色固体, 产率 73\%. m.p. 95 97 ${ }^{\circ} \mathrm{C} ;{ }^{1} \mathrm{H} \mathrm{NMR}\left(\mathrm{CDCl}_{3}, 400 \mathrm{MHz}\right)$ $\delta: 8.15(\mathrm{~d}, J=8.40 \mathrm{~Hz}, 1 \mathrm{H}), 7.84(\mathrm{~s}, 1 \mathrm{H}), 7.73 \sim 7.76(\mathrm{~m}$, $3 \mathrm{H}), 7.43 \sim 7.58(\mathrm{~m}, 4 \mathrm{H}), 6.83(\mathrm{~s}, 4 \mathrm{H}), 5.11(\mathrm{~s}, 2 \mathrm{H}), 3.75$ $(\mathrm{s}, 3 \mathrm{H}), 3.61(\mathrm{~s}, 3 \mathrm{H}), 2.37(\mathrm{~s}, 3 \mathrm{H}) ;{ }^{13} \mathrm{C} \mathrm{NMR}\left(\mathrm{CDCl}_{3}, 100\right.$ MHz) $\delta: 155.78,150.62,148.46,146.84,146.51,141.23$, $138.55,136.42,132.25,129.84,128.25,124.40,122.65$, $120.33,116.33,114.92,110.36,99.79,75.16,55.67,34.19$, 
14.78. Anal. calcd for $\mathrm{C}_{26} \mathrm{H}_{24} \mathrm{~N}_{6} \mathrm{O}_{3}$ : C 66.65, $\mathrm{H}$ 5.16, $\mathrm{N}$ 17.94; found C 66.80, H 5.06, N 17.80.

$O$-[4-(1H-苯并三唑-1-基)苄基]-5-(4-三氟甲氧基苯 氧基)-3-甲基-1-甲基- $1 H$-吡唑-4-甲酰基肟(7j): 无色油 状物, 产率 $71 \%$. ${ }^{1} \mathrm{H}$ NMR $\left(\mathrm{CDCl}_{3}, 400 \mathrm{MHz}\right) \delta: 8.16(\mathrm{~d}$, $J=8.40 \mathrm{~Hz}, 1 \mathrm{H}), 7.86(\mathrm{~s}, 1 \mathrm{H}), 7.73 \sim 7.76(\mathrm{~m}, 3 \mathrm{H}), 7.43 \sim$ $7.58(\mathrm{~m}, 4 \mathrm{H}), 7.18$ (d, $J=8.80 \mathrm{~Hz}, 2 \mathrm{H}), 6.91$ (d, $J=8.80$ $\mathrm{Hz}, 2 \mathrm{H}), 5.07$ (s, 2H), 3.62 (s, 3H), 2.37 (s, 3H); ${ }^{13} \mathrm{C}$ NMR $\left(\mathrm{CDCl}_{3}, 100 \mathrm{MHz}\right) \delta: 154.91,147.15,147.08,146.53$, $144.78,140.68,138.35,136.51,132.25,129.79,128.28$, $124.42,122.87,121.68$ (q, $J=255.50 \mathrm{~Hz}), 116.37,116.16$, $110.32,100.17,75.24,34.27$, 14.49. Anal. calcd for $\mathrm{C}_{26} \mathrm{H}_{21} \mathrm{~F}_{3} \mathrm{~N}_{6} \mathrm{O}_{3}$ : C 59.77, H 4.05, N 16.08; found C 59.91, $\mathrm{H}$ 4.25, N 16.01 .

$O$-[4-(1H-苯并三唑-1-基)芐基]-5-(3-甲基苯氧基)-3甲基-1-甲基- $1 H$-吡唑-4-甲酰基肟(7k)：白色固体，产率 69\%. m.p. 103 105 ${ }^{\circ} \mathrm{C} ;{ }^{1} \mathrm{H}$ NMR $\left(\mathrm{CDCl}_{3}, 400 \mathrm{MHz}\right) \delta$ : $8.15(\mathrm{~d}, J=8.40 \mathrm{~Hz}, 1 \mathrm{H}), 7.86(\mathrm{~s}, 1 \mathrm{H}), 7.73$ (d, $J=8.40$ $\mathrm{Hz}, 3 \mathrm{H}), 7.43 \sim 7.58(\mathrm{~m}, 4 \mathrm{H}), 6.67 \sim 7.21(\mathrm{~m}, 4 \mathrm{H}), 5.12(\mathrm{~s}$, 2H), $3.60(\mathrm{~s}, 3 \mathrm{H}), 2.39(\mathrm{~s}, 3 \mathrm{H}), 2.31(\mathrm{~s}, 3 \mathrm{H}) ;{ }^{13} \mathrm{C} \mathrm{NMR}$ $\left(\mathrm{CDCl}_{3}, 100 \mathrm{MHz}\right) \delta: 156.73,147.94,146.83,146.50$, $141.22,140.37,138.52,136.42,132.26,129.86,129.71$, $128.24,124.51,124.39,122.67,120.34,115.83,112.24$, $110.34,100.18,75.18,34.23,21.43,14.83$. Anal. calcd for $\mathrm{C}_{26} \mathrm{H}_{24} \mathrm{~N}_{6} \mathrm{O}_{2}$ : C 69.01, H 5.35, N 18.57; found $\mathrm{C} 68.82, \mathrm{H}$ $5.52, \mathrm{~N} 18.43$.

$O$-[4-(1H-苯并三唑-1-基)芐基]-5-(4-甲基苯氧基)-3甲基-1-甲基- $1 H$-吡唑-4-甲酰基肟(71): 白色固体, 产率 75\%. m.p. 91 93 ${ }^{\circ} \mathrm{C} ;{ }^{1} \mathrm{H}$ NMR $\left(\mathrm{CDCl}_{3}, 400 \mathrm{MHz}\right) \delta$ : $8.15(\mathrm{~d}, J=8.00 \mathrm{~Hz}, 1 \mathrm{H}), 7.85(\mathrm{~s}, 1 \mathrm{H}), 7.72 \sim 7.75(\mathrm{~m}$, $3 \mathrm{H}), 7.53 \sim 7.58(\mathrm{~m}, 3 \mathrm{H}), 7.42 \sim 7.46(\mathrm{~m}, 1 \mathrm{H}), 7.10(\mathrm{~d}, J=$ $8.40 \mathrm{~Hz}, 2 \mathrm{H}), 6.79$ (d, J=8.80 Hz, 2H), 5.11 (s, 2H), 3.60 $(\mathrm{s}, 3 \mathrm{H}), 2.38(\mathrm{~s}, 3 \mathrm{H}), 2.29(\mathrm{~s}, 3 \mathrm{H}) ;{ }^{13} \mathrm{C} \mathrm{NMR}\left(\mathrm{CDCl}_{3}, 100\right.$ MHz) $\delta: 154.70,148.16,146.85,146.53,141.26,138.57$, $136.44,133.24,132.29,130.47,129.89,128.27,124.42$, 122.69 , 120.38, 115.11, 110.36, 100.09, 75.19, 34.23, 20.58, 14.84. Anal. calcd for $\mathrm{C}_{26} \mathrm{H}_{24} \mathrm{~N}_{6} \mathrm{O}_{2}$ : C 69.01, H 5.35, N 18.57; found C 69.18, H 5.20, N 18.50.

$O$-[4-(1H-苯并三唑-1-基)芐基]-5-(4-叔丁基苯氧 基)-3-甲基-1-甲基- $1 H$-吡唑-4-甲酰基肟(7m)：无色油状 物, 产率 $68 \% .{ }^{1} \mathrm{H} \mathrm{NMR}\left(\mathrm{CDCl}_{3}, 400 \mathrm{MHz}\right) \delta: 8.15(\mathrm{~d}, J=$ $8.40 \mathrm{~Hz}, 1 \mathrm{H}), 7.86(\mathrm{~s}, 1 \mathrm{H}), 7.73 \sim 7.76(\mathrm{~m}, 3 \mathrm{H}), 7.42 \sim$ 7.57 (m, 4H), 7.32 (d, $J=8.80 \mathrm{~Hz}, 2 \mathrm{H}), 6.82$ (d, $J=8.80$ $\mathrm{Hz}, 2 \mathrm{H}), 5.11$ (s, 2H), 3.61 (s, 3H), 2.40 (s, 3H), 1.29 (s, $9 \mathrm{H}) ;{ }^{13} \mathrm{C} \mathrm{NMR}\left(\mathrm{CDCl}_{3}, 100 \mathrm{MHz}\right) \delta: 154.53,148.22$,
$146.79,146.62,146.50,141.27,138.49,136.45,132.27$, $129.89,129.38,128.25,126.79,124.39,122.71,120.34$, 114.72, 110.33, 100.10, 75.19, 34.29, 34.23, 31.41, 14.92. Anal. calcd for $\mathrm{C}_{29} \mathrm{H}_{30} \mathrm{~N}_{6} \mathrm{O}_{2}$ : C 70.42, H 6.11, N 16.99; found $\mathrm{C}$ 70.26, H 6.30, N 16.86 .

$O$-[4-(1H-苯并三唑-1-基) 茮基]-5-(2,3-二氟苯氧 基)-3-甲基-1-甲基- $1 H$-吡唑-4-甲酰基肟(7n)：白色固体， 产率 63\%. m.p. $109 \sim 111{ }^{\circ} \mathrm{C} ;{ }^{1} \mathrm{H}$ NMR $\left(\mathrm{CDCl}_{3}, 400\right.$ MHz) $\delta: 8.16(\mathrm{~d}, J=8.00 \mathrm{~Hz}, 1 \mathrm{H}), 7.87(\mathrm{~s}, 1 \mathrm{H}), 7.73 \sim$ $7.76(\mathrm{~m}, 3 \mathrm{H}), 7.54 \sim 7.58(\mathrm{~m}, 1 \mathrm{H}), 7.51(\mathrm{~d}, J=8.40 \mathrm{~Hz}$, $2 \mathrm{H}), 7.43 \sim 7.47(\mathrm{~m}, 1 \mathrm{H}), 6.89 \sim 6.94(\mathrm{~m}, 2 \mathrm{H}), 6.50 \sim 6.54$ $(\mathrm{m}, 1 \mathrm{H}), 5.06(\mathrm{~s}, 2 \mathrm{H}), 3.67$ (s, 3H), $2.35(\mathrm{~s}, 3 \mathrm{H}) ;{ }^{13} \mathrm{C} \mathrm{NMR}$ $\left(\mathrm{CDCl}_{3}, 100 \mathrm{MHz}\right) \delta: 151.56(\mathrm{~d}, J=247.70 \mathrm{~Hz}), 147.10$, $146.66,146.51,145.66,142.43,140.43,139.94,138.48$, $136.41,132.28,129.59,128.28,124.42,123.48,122.70$, $120.35,112.25$ (d, $J=17.30 \mathrm{~Hz}), 111.47,110.34,99.92$, 75.17, 34.26, 14.19. Anal. calcd for $\mathrm{C}_{25} \mathrm{H}_{20} \mathrm{~F}_{2} \mathrm{~N}_{6} \mathrm{O}_{2}$ : C 63.29, H 4.25, N 17.71; found C 63.15, H 4.09, N 17.82.

$O$-[4-(1H-苯并三唑-1-基) 苦基]-5-(2,4-二氯苯氧 基)-3-甲基-1-甲基- $1 H$-吡唑-4-甲酰基肜(7o)：白色固体， 产率 66\%. m.p. $121 \sim 123{ }^{\circ} \mathrm{C} ;{ }^{1} \mathrm{H}$ NMR $\left(\mathrm{CDCl}_{3}, 400\right.$ $\mathrm{MHz}) \delta: 8.16(\mathrm{~d}, J=8.40 \mathrm{~Hz}, 1 \mathrm{H}), 7.85(\mathrm{~s}, 1 \mathrm{H}), 7.74 \sim$ $7.76(\mathrm{~m}, 3 \mathrm{H}), 7.56 \sim 7.58(\mathrm{~m}, 1 \mathrm{H}), 7.49(\mathrm{~d}, J=8.40 \mathrm{~Hz}$, $2 \mathrm{H}), 7.43 \sim 7.47(\mathrm{~m}, 2 \mathrm{H}), 7.09 \sim 7.12(\mathrm{~m}, 1 \mathrm{H}), 6.61(\mathrm{~d}, J=$ $8.80 \mathrm{~Hz}, 1 \mathrm{H}), 5.06(\mathrm{~s}, 2 \mathrm{H}), 3.65(\mathrm{~s}, 3 \mathrm{H}), 2.35(\mathrm{~s}, 3 \mathrm{H}) ;{ }^{13} \mathrm{C}$ NMR $\left(\mathrm{CDCl}_{3}, 100 \mathrm{MHz}\right) \delta: 150.87,147.17,146.56$, $146.52,140.37,138.40,136.46,132.25,130.55,129.68$, $129.07,128.26,127.90,124.40,123.60,122.65,120.36$, $116.23,110.35,100.02,75.22,34.26,14.18$. Anal. calcd

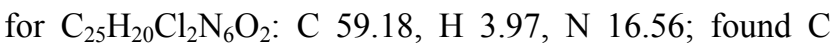
59.30, H 3.80, N 16.38 .

$O$-[4-(1H-苯并三唑-1-基)苠基]-5-(4-氟苯氧基)-3-甲 基-1-(4-甲基苯基)- $1 H$-吡唑-4-甲酰基肟(7p)：白色固体， 产率 62\%. m.p. $116 \sim 118{ }^{\circ} \mathrm{C} ;{ }^{1} \mathrm{H}$ NMR $\left(\mathrm{CDCl}_{3}, 400\right.$ $\mathrm{MHz}) \delta: 8.16(\mathrm{~d}, J=8.40 \mathrm{~Hz}, 1 \mathrm{H}), 7.90(\mathrm{~s}, 1 \mathrm{H}), 7.74 \sim$ $7.77(\mathrm{~m}, 3 \mathrm{H}), 7.53 \sim 7.58(\mathrm{~m}, 3 \mathrm{H}), 7.43 \sim 7.46(\mathrm{~m}, 3 \mathrm{H})$, $7.16(\mathrm{~d}, J=8.00 \mathrm{~Hz}, 2 \mathrm{H}), 6.92 \sim 6.96(\mathrm{~m}, 2 \mathrm{H}), 6.83 \sim 6.87$ (m, 2H), $5.12(\mathrm{~s}, 2 \mathrm{H}), 2.46(\mathrm{~s}, 3 \mathrm{H}), 2.33(\mathrm{~s}, 3 \mathrm{H}) ;{ }^{13} \mathrm{C}$ NMR $\left(\mathrm{CDCl}_{3}, 100 \mathrm{MHz}\right) \delta: 158.72(\mathrm{~d}, J=240.00 \mathrm{~Hz}), 152.66$, $148.23,147.13,146.53,140.81,138.43,137.40,136.53$, $134.94,132.29,129.82,129.75,128.31,124.45,122.73$, 122.23, 120.39, 116.72, 116.46 (d, $J=23.00 \mathrm{~Hz}$ ), 110.36, 101.58, 75.33, 21.06, 14.90. Anal. calcd for $\mathrm{C}_{31} \mathrm{H}_{25} \mathrm{FN}_{6} \mathrm{O}_{2}$ : C 69.91, H 4.73, N 15.78; found C 69.72, H 4.86, N 15.89 .

$O$-[4-(1H-苯并三唑-1-基)芐基]-5-(4-甲基苯氧基)-3- 
甲基-1-(4-甲基苯基)- $1 H$-吡唑-4-甲酰基肜(7q)：白色固 体, 产率 65\%. m.p. 103 105 ${ }^{\circ} \mathrm{C} ;{ }^{1} \mathrm{H}$ NMR $\left(\mathrm{CDCl}_{3}, 400\right.$ $\mathrm{MHz}) \delta: 8.15(\mathrm{~d}, J=8.40 \mathrm{~Hz}, 1 \mathrm{H}), 7.88(\mathrm{~s}, 1 \mathrm{H}), 7.75(\mathrm{~d}$, $J=8.40 \mathrm{~Hz}, 3 \mathrm{H}), 7.54 \sim 7.57(\mathrm{~m}, 3 \mathrm{H}), 7.42 \sim 7.47(\mathrm{~m}, 3 \mathrm{H})$, 7.15 (d, $J=8.40 \mathrm{~Hz}, 2 \mathrm{H}), 7.05(\mathrm{~d}, J=8.40 \mathrm{~Hz}, 2 \mathrm{H}), 6.79$ (d, $J=8.80 \mathrm{~Hz}, 2 \mathrm{H}), 5.14$ (s, 2H), 2.47 (s, 3H), 2.32 (s, $3 \mathrm{H}), 2.25(\mathrm{~s}, 3 \mathrm{H}) ;{ }^{13} \mathrm{C} \mathrm{NMR}\left(\mathrm{CDCl}_{3}, 100 \mathrm{MHz}\right) \delta: 154.72$, $148.07,147.50,146.49,141.09,138.49,137.12,136.44$, $135.02,133.14,132.26,130.35,129.85,129.66,128.26$, $124.41,122.68,122.11,120.34,115.28,110.34,101.56$, 75.26, 21.01, 20.55, 15.07. Anal. calcd for $\mathrm{C}_{32} \mathrm{H}_{28} \mathrm{~N}_{6} \mathrm{O}_{2}$ : C 72.71, H 5.34, N 15.90; found C 72.55, H 5.16, N 15.98.

辅助材料(Supporting Information) 目标化合物 $7 \mathbf{a} \sim$ $7 \mathbf{q}$ 的核磁共振氢谱与碳谱共振图谱. 这些材料可以免 费从本刊网站(http://sioc-journal.cn/)上下载.

\section{References}

[1] Wang, X.; Li, P.; Li, Z. N.; Yin, J.; He, M.; Xue, W.; Chen, Z. W.; Song, B. A. J. Agric. Food Chem. 2013, 61, 9575.

[2] Liu, Y. X.; Song, H. J.; Huang, Y. Q.; Li, J. R.; Zhao, S.; Song, Y. C.; Yang, P. W.; Xiao, Z. X.; Liu, Y. X.; Li, Y. Q.; Shang, H.; Wang, Q. M. J. Agric. Food Chem. 2014, 62, 9987.

[3] Gan, X. H.; Hu, D. Y.; Li, P.; Wu, J.; Chen, X. W.; Xue, W. Song, B. A. Pest. Manage. Sci. 2016, 72, 534.

[4] Kumar, K. S.; Ramulu, M. S.; Rajesham, B.; Kumar, N. P.; Voora, V.; Kancha, R. K. Org. Biomol. Chem. 2017, 15, 4468.

[5] Li, P.; Hu, D. Y.; Xie, D. D.; Chen, J. X.; Jin, L. H.; Song, B. A. J. Agric. Food Chem. 2018, 66, 3093.

[6] Yuan, Y.; Subedi, L.; Lim, D.; Jung, J. K.; Kim, S. Y.; Seo, S. Y. Bioorg. Med. Chem. Lett. 2019, 29, 329.

[7] Facchetti, G.; Rimoldi, I. Bioorg. Med. Chem. Lett. 2019, 29, 1257.

[8] Zhu, X. L.; Zhang, R.; Wu, Q. Y.; Song, Y. J.; Wang, Y. X.; Yang, J. F.; Yang, G. F. J. Agric. Food Chem. 2019, 67, 2774.

[9] Prandi, C.; Occhiato, E. G. Pest. Manage. Sci. 2019, 75, 2385.

[10] Zeng, D.; Wang, M. W.; Xiang, M.; Liu, L. W.; Wang, P. Y.; Li, Z.; Yang, S. Pest. Manage. Sci. 2020, 76, 2681.

[11] He, F. Q.; Wang, B. L.; Li, Z. M.; Song, H. B. Chin. J. Struct. Chem. 2006, 25, 543.

[12] Zhang, S. S.; Wan, J.; Li, X. M.; Li, C. L.; Xu, L. Z. Chem. Res. Chin. Univ. 2007, 23, 120

[13] Fu, J.; Yang, Y.; Zhang, X. W.; Mao, W. J.; Zhang, Z. M.; Zhu, H. L. Bioorg. Med. Chem. 2010, 18, 8457.
[14] Wan, J.; Lü, P. C.; Tian, N. N.; Zhu, H. L. J. Chem. Sci. 2010, 122, 597.

[15] Mansour, A. M.; Mohamed, M. F. Inorg. Chim. Acta 2014, 423, 373.

[16] He, Z.; Zhang, S. F.; Xue, J. R.; Liang, Y.; Zhang, X.; Jin, L. H.; Qin, D. B. J. Organomet. Chem. 2016, 808, 12.

[17] Wan, J.; Yan, X.; Ma, C. P.; Bi, S.; Zhu, H. L. Med. Chem. Res. 2010, 19, 970 .

[18] He, F. Q.; Liu, X. H.; Wang, B. L.; Li, Z. M. J. Chem. Res. 2006, 12,809 .

[19] Ouyang, G. P.; Chen, Z.; Cai, X. J.; Song, B. A.; Bhadury, P. S.; Yang, S.; Jin, L. H.; Xue, W.; Hu, D. Y.; Zeng, S. Bioorg. Med. Chem. 2008, 16, 9699.

[20] Dai, H.; Ye, L. Y.; Zhuang, H. Y.; Dai, B. J.; Fang, Y.; Shi, Y. J. Molecules 2015, 20, 21870.

[21] Dai, H.; Yao, W.; Sun, S. Y.; Li, L.; Shi, L.; Qian, H. W.; Li, C. J.; Shi, J.; Shi, Y. J. Chin. J. Org. Chem. 2017, 37, 3155 (in Chinese). (戴红，姚炜，孙思宇，李玲，施磊，钱宏炜，李春建，石健，石 玉军, 有机化学, 2017, 37, 3155.)

[22] Xiong, B.; Chen, S.; Zhu, P.; Huang, M. L.; Gao, W. J.; Zhu, R.; Qian, J. Q.; Peng, Y. F.; Zhang, Y. A.; Dai, H.; Ling, Y. Med. Chem. 2019, 15, 743.

[23] Swanson, M. B.; Ivancic, W. A.; Saxena, A. M.; Allton, J. D.; O’Brien, G. K.; Suzuki, T.; Nishizawa, H.; Nokata, M. J. Agric. Food Chem. 1995, 43, 513.

[24] Fu, C. R.; Peng, J.; Ning, Y.; Liu, M.; Shan, P. C.; Liu, J.; Li, Y. Q.; Hu, F. Z.; Zhu, Y. Q.; Yang, H. Z.; Zou, X. M. Pest. Manage. Sci. 2014, 70, 1207.

[25] Zhou, Q.; Zheng, D. D.; Shi, Y. J.; Yao, W.; Qian, H. W.; Ding, Y.; Wei, Z. H.; Shen, A. B.; Feng, X.; Shi, J.; Dai, H. Chin. J. Org. Chem. 2018, 38, 3318 (in Chinese).

(周钱, 郑丹丹, 石玉军, 姚炜，钱宏炜，丁颖，魏中吴，沈爱宝， 冯霞, 石健, 戴红, 有机化学, 2018, 38, 3318.)

[26] Wang, Y.; Ye, H.; Qian, C.; Yao, W.; Li, H.; Wang, K.; Hu, L. P.; Li, L.; Wu, J. M.; Dai, H. Chin. J. Org. Chem. 2020, 40, 232 (in Chinese).

(王杨, 叶浩, 钱程, 姚炜, 李宏, 王凯, 胡兰萍, 李玲, 吴锦明, 戴红, 有机化学, 2020, 40, 232.)

[27] Dai, H.; Yao, W.; Fang, Y.; Sun, S. Y.; Shi, Y. J.; Chen, J.; Jiang, G. Q.; Shi, J. Molecules 2017, 22, 2000.

[28] Wang, Y.; Ye, H.; Qian, C.; Yao, W.; Li, H.; Wang, K.; Hu, L. P.; Li, L.; Wu, J. M.; Dai, H. Chin. J. Org. Chem. 2020, 40, 528 (in Chinese).

(丁成荣, 潘亚运, 谭成侠, 有机化学, 2020, 40, 528.)

[29] Tanaka, A.; Terasawa, T.; Hagihara, H.; Sakuma, Y.; Ishibe, N.; Sawada, M.; Takasugi, H.; Tanaka, H. J. Med. Chem. 1998, 41, 2390.

[30] Dai, H.; Huang, M. L.; Qian, J. Q.; Liu, J.; Meng, C.; Li, Y. Y.; Ming, G. X.; Zhang, T.; Wang, S. L.; Shi, Y. J.; Yao, Y.; Ge, S. S.; Zhang, Y. A.; Ling, Y. Eur. J. Med. Chem. 2019, 166, 470. 